\title{
Les humanités, la culture humaniste et la culture scolaire
}

Nathalie Denizot

\section{(2) OpenEdition}

1 Journals

\section{Édition électronique}

URL : http://journals.openedition.org/trema/3301

DOI : 10.4000/trema.3301

ISSN : 2107-0997

\section{Éditeur}

Faculté d'Éducation de l'université de Montpellier

\section{Édition imprimée}

Date de publication : 1 mai 2015

Pagination : $42-51$

ISSN : 1167-315X

\section{Référence électronique}

Nathalie Denizot, «Les humanités, la culture humaniste et la culture scolaire », Tréma [En ligne], 43

2015, mis en ligne le 25 juin 2015, consulté le 10 décembre 2020. URL : http://

journals.openedition.org/trema/3301; DOI : https://doi.org/10.4000/trema.3301

Ce document a été généré automatiquement le 10 décembre 2020.

Trema 


\title{
Les humanités, la culture humaniste et la culture scolaire
}

\author{
Nathalie Denizot
}

1 Longtemps suspectes et tenues à distance, les humanités semblent refaire depuis une ou deux décennies un retour en force : les universités réinventent des cursus d'humanités ${ }^{1}$, la première définition du socle commun (celle de $2006^{2}$ ), cadre de référence pour la scolarité obligatoire en France ${ }^{3}$, fait de la "culture humaniste " sa cinquième grande compétence, et plusieurs ouvrages récents ont également contribué à les revivifier, sans verser dans la nostalgie (par exemple Citton, 2010 ; Nussbaum, 2010 ; Doueihi, 2011). Ce retour, porté sans doute également par la traduction du terme anglais "humanities", très usuel dans le monde anglophone, s'accompagne de réflexions sur l'avenir de nos sociétés et de nos cultures. Plus modestement, je voudrais ici interroger le retour de la notion à l'école et dans l'enseignement supérieur, pour comprendre ce qu'elle emprunte réellement au passé et comment elle s'articule - ou non - avec la culture scolaire et avec les disciplines.

\section{Les humanités : un système d'éducation et d'organisation de la forme scolaire.}

Mon propos n'est pas ici de revenir précisément sur ce que furent les humanités, dont l'histoire a fait l'objet de travaux importants (Falcucci, 1939 ; Compère et Chervel, 1997 ; Compère, 1997; Chervel, 2006; Laurenti et Vignest, 2010 ; etc.). Je me contenterai de rappeler brièvement quelques-unes des caractéristiques essentielles de ces humanités qu'on n'appela «classiques » que lorsqu'elles commencèrent à être ébranlées, pour essayer ensuite de mieux cerner ce qu'elles deviennent dans l'institution scolaire et/ou universitaire actuelle.

Dans son sens le plus répandu ${ }^{4}$, on désigne par humanités, depuis l'Ancien régime, ce qu'on enseigne dans les collèges autour du latin et des auteurs latins essentiellement (le grec est en réalité relativement peu présent, et quand il l'est, c'est davantage à travers 
des textes qu'à travers sa langue). Comme le rappellent Marie-Madeleine Compère et André Chervel (1997, p. 6) :

Dans l'enseignement français traditionnel, les humanités classiques se définissent d'abord et surtout par une "éducation », une éducation esthétique, rhétorique, mais également morale et civique.

C'est donc une formation complète et "gratuite ", qui vise une éducation libérale (au sens de former des hommes libres) et morale. Le latin signe symboliquement l'appartenance à une élite et l'exercice roi est le discours latin, qui vient parachever l'enseignement rhétorique. Plus qu'une "discipline", les humanités forment un système éducatif, caractérisé par ce que Renée Balibar (1993) nomme le colinguisme, associant plusieurs langues écrites (le latin et le français, et à la marge le grec) : c'est le mode des relations des langues entre elles qui fait tenir l'édifice des humanités classiques. Et si les auteurs français y sont présents, ils le sont comme traducteurs et imitateurs des auteurs antiques, avec qui ils forment ce que Chervel (2006, p. 424) appelle des "couples canoniques" (Boileau et Horace, Bossuet et Démosthène, La Fontaine et Ésope et Phèdre, Mme de Sévigné et Pline Le Jeune, etc.).

5 La contestation des humanités, qui commence dès le milieu du XVIIIe siècle (Chervel et Compère, 1997, p. 27), n'a eu de cesse tout au long du XIXe siècle. Tout d'abord parce que la langue et la littérature françaises prennent de plus en plus d'importance : en 1840 est instaurée l'explication d'auteur français au bac, et la seconde moitié du siècle voit la montée en puissance de l'historicisation de la littérature et de l'enseignement de l'histoire de la littérature française, installé dès 1863 dans l'enseignement secondaire spécial. La diversification de l'enseignement secondaire (plan Fortoul en 1852, création par Victor Duruy de l'enseignement secondaire spécial en 1863) joue d'ailleurs un rôle essentiel dans la remise en question des humanités. Martine Jey (1998) a bien montré en effet combien les filières dominées (secondaire spécial, enseignement féminin) ont fonctionné comme des laboratoires de réformes et d'évolutions. La part de la langue et de la littérature française y était plus importante que dans l'enseignement secondaire classique (exclusivement masculin jusqu'en 1924, année de fusion des programmes des deux enseignements), puisque ces filières étaient des filières sans latin, et qu'il avait fallu inventer une nouvelle discipline et un nouveau rapport à la langue et aux textes. C'est aussi dans l'enseignement spécial que la part des sciences est la plus importante dans la deuxième moitié du XIXe siècle (cf. Belhoste, 1990), avant la réforme de 1902 qui consacre un enseignement « moderne » - c'est-à-dire sans latin et avec une part importante pour les enseignements scientifiques - face à l'enseignement «classique» héritier des anciennes humanités.

Ce sont donc les réformes de 1902 qui sonnent le glas des humanités classiques, en instituant quatre filières ${ }^{5}$, dont une sans latin. Après un siècle de polémiques, c'est une réforme de structure, et non pas simplement de programme, qui donne le coup de grâce aux humanités classiques et laisse place à d'autres "humanités", scientifiques ou modernes. Cette mutation du modèle éducatif classique vers le modèle moderne n'est pas, comme le soulignent Chervel et Compère (1997, p. 34), un « simple changement de gravité disciplinaire ». De nombreuses matières (histoire, poétique, rhétorique mais aussi géographie ancienne, mythologie, etc.) concouraient en effet à la formation humaniste et toutes relevaient de la compétence du même maitre: «leur trait commun, c'est qu'en aucun cas, à la différence des langues étrangères ou des mathématiques, elles ne pouvaient constituer des enseignements indépendants » (id.). Le nouveau modèle marque 
au contraire l'autonomisation de disciplines comme l'histoire ou la littérature. Et l'une des conséquences concrètes et marquantes de cette mutation concerne l'organisation des journées : on passe de la « classe » de deux heures qui occupait chaque demi-journée à des classes d'une heure, réparties entre les diverses disciplines. La fin des humanités comme système d'enseignement est ainsi liée à des modifications importantes dans la forme scolaire elle-même.

\section{De nouvelles humanités}

7 Cela dit, il y a là une forme de paradoxe: si les humanités classiques sont fortement marginalisées par la diversification des filières, et vidées en grande partie de leur substance par la disparition du discours latin, elles ne disparaissent pas complètement. D'ailleurs, comme le rappelle Dan Savatovsky (1995, p. 53), le français (en tant que discipline scolaire) «ne s'est pas imposé contre les humanités : il en est largement le produit ». La contestation des humanités vise davantage à tenter de les refonder qu'à les supprimer. C'était déjà le cas dans l'enseignement spécial, dont l'effort, écrit Clément Falcucci (1939, p. 294) «a consisté, en définitive, à dégager de nouvelles "humanités" ». Quant aux responsables républicains de l'Instruction publique des années 1880, ils formulèrent l'idée d'«humanités primaires », «idée inouïe jusqu'alors", comme le rappelle Pierre Kahn (2001, p. 47), tant l'idée d'une formation libérale était associée à l'enseignement secondaire, l'enseignement primaire restant longtemps cantonné à l'apprentissage de fondamentaux dans une conception utilitaire.

8 La refondation des humanités passe donc par de nouvelles humanités, mais si les expressions « humanités scientifiques » ou « humanités modernes » co-existent aux côtés des humanités rebaptisés "classiques", il faut noter que cette extension de l'expression marque des changements importants dans le paradigme des humanités. D'ailleurs, comme le signale Jey (1998, p. 183), la querelle qui opposa autour de la réforme de 1902 les « Anciens » et les « Modernes » se joua aussi autour des mots, et les Anciens refusèrent d'employer les expressions "d'humanités françaises, ou d'humanités modernes, [...] utilisées par les partisans de l'enseignement moderne » : ajouter une expansion modifie le sens et les connotations du mot. Les humanités modernes, à visée de culture générale, remplacent certes les langues anciennes par les langues vivantes, mais sans réinstaurer de colinguisme. Elles inventent ainsi une nouvelle discipline, le français, organisé autour de la littérature française, et autour de nouveaux exercices et de nouvelles pratiques de lecture et d'écriture. Quant aux humanités scientifiques, elles instaurent une nouvelle " conception du monde fondée sur la connaissance des lois de la matière " (Chervel et Compère, 1997, p. 31) et, en réalité, un nouveau rapport aux textes, à la langue, à la morale. Et le terme a encore bougé pour accompagner les nouvelles « humanités » qui ont vu le jour au cours du XXe siècle : des humanités techniques ${ }^{6}$ ou professionnelles (cf. le système scolaire belge et ses quatre catégories d'humanités : générales, technologiques, techniques, professionnelles); et surtout des humanités numériques (de l'anglais Digital Humanities) qui ne se contentent plus de désigner la numérisation de contenus, mais qui peuvent aller jusqu'à se revendiquer d'un « humanisme numérique » (Doueihi, 2011).

$9 \quad$ S'agit-il d'une dilution de la notion, ou au contraire d'une extension de la notion ? Il est souvent difficile de trancher, d'autant qu'il n'est pas toujours clair non plus de savoir si ces nouveaux emplois sont simplement métaphoriques ou s'ils reconstruisent véritablement la notion. En tout cas, ce n'est qu'en déplaçant leur champ de légitimité 
que les humanités survivent. Et ce qui ressort de ces nouvelles acceptions du terme ${ }^{7}, c^{\prime}$ est la mise à mal de la prétention des humanités à l'universalisme: si ces nouvelles humanités contribuent à rajeunir la notion, elles poursuivent en même temps l'entreprise de relativisation amorcée dès l'ajout de l'adjectif «classiques » à ce qui était jusqu'alors conçu comme une entité quasi absolue.

\section{Le retour des humanités}

Dans le champ de l'enseignement, le terme "humanités » semble réapparaitre depuis quelques années, mais avec des acceptions qui ne sont pas complètement stabilisées. Il est présent dans l'enseignement supérieur pour désigner généralement (à la manière des humanities anglo-saxonnes) un pôle pluridisciplinaire ou un ensemble de disciplines, articulés autour des lettres (classiques et modernes), des langues et de la philosophie. Un certain nombre de maquettes universitaires (y compris dans les $\mathrm{ESPE}^{8}$ ) utilisent ainsi l'expression pour désigner la partie des enseignements qui correspond à ces domaines. Et dans l'enseignement secondaire, où le terme ${ }^{9}$ est quasiment absent, on le trouve cependant dans les programmes de première et terminale L de 2010, comme synonyme d'une filière post-bac « littéraire » élargie :

L'enseignement spécifique de littérature à destination des élèves de la série L doit permettre un approfondissement et un élargissement des connaissances nécessaires à :

- la construction et à la consolidation de leur culture littéraire ;

- la découverte de problématiques liées à la poursuite d'études dans le champ des humanités.

11 Mais l'empan disciplinaire des humanités n'est pas véritablement défini, et le terme est parfois utilisé plus largement encore. L'Université de Lille 3 a récemment réorganisé certaines UFR ${ }^{10}$ et créé une UFR baptisée « Humanités » qui regroupe, en plus des lettres et de la philosophie, les arts et les sciences du langage ; à Bordeaux 3, l'UFR Humanités comprend également un département Histoire et un département Histoire de l'art et archéologie ; quant à l'École doctorale " des humanités » de l'Université de Haute-Alsace, elle englobe même les sciences de l'éducation et les sciences de la communication, aux côtés de l'archéologie industrielle ou de la littérature, entre autres.

Cette autonomisation d'un "champ des humanités", pour reprendre l'expression du programme de lycée, est intéressante à plus d'un titre, par les questions qu'elle soulève : les humanités sont-elles un champ de recomposition de disciplines non "scientifiques", et qui permettrait d'effacer ou de subsumer les tensions et/ou distinctions entre, par exemple, linguistique et littérature, histoire et économie, littérature et philosophie, etc. ? S'agit-il d'intégrer les arts - au risque d'une disciplinarisation des arts dans la forme scripturo-scolaire des savoirs que décrit Lahire (2008), et aux dépens des pratiques (les pratiques artistiques restant par exemple du côté des écoles d'art)? Et enfin, quelle articulation de ce champ avec celui des "sciences humaines", qui regroupe des disciplines (comme l'histoire) parfois annexées par les humanités? Autant de questions auxquelles les recompositions actuelles ne permettent pas forcément de répondre, et qui ne semblent d'ailleurs pas pensées, tant ces rapprochements disciplinaires semblent se faire de manière plus administrative que scientifique (il n'y a pas nécessairement de rapprochement des laboratoires, par exemple). Comme le souligne Jean-Louis Fabiani 
(2012, p. 139), «l'objectivation de nos statuts disciplinaires est d'autant plus difficile qu'elle menace de mettre en question la stabilité de nos arrangements institutionnels ».

\section{Culture humaniste, socle commun et culture scolaire}

13 Il est par ailleurs impossible de réfléchir actuellement aux « humanités » sans prendre en compte ce qu'on appelle la "culture humaniste ", les deux expressions ne se recouvrant pas totalement, et la seconde étant plus spécifique à l'enseignement primaire et secondaire. Ce qui m'intéresse est ici d'essayer d'explorer comment cette culture humaniste s'articule à la culture scolaire, entendue, comme le propose Chervel (2005) comme une culture originale, autonome (et non simplement comme la culture acquise à l'école ni la partie de la culture des adultes que transmet l'école): quels rapports la culture humaniste entretient-elle avec les finalités de l'école (éduquer/instruire) ? avec les contenus scolaires, notamment avec les corpus de textes? voire avec la forme scolaire? Questions d'autant plus complexes que la culture scolaire est en tension entre des formes de stabilité (cf. l'importance du patrimoine ou de la tradition scolaire) et d'incessantes recompositions : l'histoire du corpus des " textes anciens " (textes antiques et mythologiques) en classe de sixième montre par exemple comment les humanités ont pu devenir un enjeu de culture générale, avant que les supplante une logique de « culture commune ", qui instrumentalise l'héritage humaniste et rebaptise les textes anciens en textes «fondateurs » (Denizot, 2013).

$14 \mathrm{Au}$ XIXe siècle, la culture humaniste correspond à ce qu'on appelle aussi la culture "classique », exprimée de manière exemplaire par les auteurs de l'antiquité, avec cette idée d'une unicité de la culture et d'un homme éternel (Lelièvre, 2009). Elle est liée à l'enseignement secondaire, et particulièrement aux classes de lettres. Or l'expression refait florès à partir de 2006, notamment depuis son institutionnalisation comme cinquième pilier du socle commun ${ }^{11}$ (2006), mais avec des différences importantes avec cette précédente acception. Tout d'abord elle ne se cantonne plus aux «humanités classiques ", l'ancrage sur les langues anciennes ayant quasiment disparu, et désigne un ensemble élargi de

"connaissances» qui font appel à la littérature, aux arts (y compris le cinéma, l'architecture, la musique, etc.), à l'histoire, à la géographie, mais aussi à des phénomènes socioculturels comme le «fait religieux » ou les droits de l'homme. Ensuite, elle a glissé des grandes classes vers les petites (le socle commun étant destiné à l'enseignement obligatoire, il correspond plutôt au primaire et au collège); enfin, elle est articulée à l'idée de la pluralité des cultures et à une forme de relativisme quant à un homme éternel. Ces différences ne sont en rien anecdotiques, et interrogent le choix de l'expression: pourquoi reprendre un terme aussi connoté pour désigner quelque chose qui n'a plus grand-chose à voir avec ce que pouvait être la « culture humaniste »?

15 Ce retour de la culture humaniste qui, comme le rappelle Lelièvre (2009), n'était plus depuis longtemps une référence pour l'école, se fait autour de cette notion de "culture ", et non autour des " humanités », terme plutôt cantonné dans le supérieur, comme je l'ai dit. Le choix des mots n'est pas anodin, et préférer « culture humaniste » à " humanités » est sans doute une manière d'évacuer la question de la rhétorique, qui n'est pas une référence scolaire habituelle pour les petites classes, mais aussi de signaler qu'il s'agit 
bien de culture, et non pas seulement de textes. C'est au prix de ce double déplacement que la culture humaniste retrouve une forme de légitimité scolaire. Mais c'est aussi en renonçant aux "humanités scientifiques»: dans le socle commun de 2006, la culture humaniste est clairement opposée à ce troisième pilier qu'est la culture scientifique, et l'on retrouve finalement ici la vieille partition entre sciences et lettres, revisitée pour ne pas laisser les lettres seules face aux sciences. De ce point de vue, la culture humaniste est bien héritière des humanités, même si l'expression peut paraitre plus moderne et plus ouverte. Et ce retour en grâce de l'humanisme comme valeur positive est à double tranchant: en effet, comme le souligne Lelièvre (2009, p. 12), l'humanisme est aussi une conception qui a favorisé une " division hiérarchisée » entre les formations intellectuelles et les formations manuelles ou techniques, conception sur laquelle repose tout notre système scolaire: il n'est pas étonnant que la culture humaniste puisse si facilement retrouver une place de choix à l'école, tant elle reste compatible avec la culture scolaire.

Il est d'ailleurs intéressant d'essayer de cerner, à travers notamment ce qu'en dit le socle commun, ce qu'on pourrait appeler la version scolaire actuelle de la culture humaniste. Une première caractéristique semble la placer dans la continuité des humanités et de l'humanisme traditionnel, puisqu'elle repose sur de "grands " textes, fondateurs ou patrimoniaux, mais sans que soient toujours très claires la manière dont on construit cette catégorie, et la distinction à opérer entre textes classiques, patrimoniaux et/ou fondateurs (Houdart-Mérot, 2012 ; Denizot, à paraitre), et sans que le corpus humaniste soit réellement interrogé. Comme le souligne Claire Doquet-Lacoste (2008, p. 99) :

Eu égard à la diversité de la population qui fréquente nos écoles, et en tenant compte de ce que les travaux des anthropologues ont montré de la racine commune à l'humanité d'un certain nombre de mythes fondateurs [...], je crois nécessaire de se demander quels mythes originels sont à enseigner, quels rapprochements seraient à faire avec des mythes non européens par exemple.

17 De même, rien n'est dit sur les pratiques de lecture de ces textes, pratiques au contraire parfaitement balisées dans les humanités classiques: il s'agit de les "fréquenter", suggère simplement le texte du socle commun, qui parle aussi de connaître les textes. On semble davantage du côté d'une forme de culture générale que des pratiques humanistes de lecture et d'écriture. D'ailleurs, cette culture humaniste est distincte de la maitrise de la langue, qui représente un pilier spécifique du socle commun, ce qui pose également la question - pourtant cruciale à l'école - de l'articulation langue et littérature. Il est intéressant également de remarquer ce qui est exclu de la sphère de la culture humaniste: outre la langue, il faut citer l'argumentation, dont il n'est que très peu question dans le socle, et jamais dans ce pilier-ci ${ }^{12}$, ce qui est d'autant plus révélateur que les pratiques et compétences argumentatives étaient essentielles dans les pratiques rhétoriques des humanités. Et surtout, toutes les disciplines scientifiques en sont exclues puisque, comme je l'ai déjà rappelé, la culture humaniste dans le socle commun est complètement distincte de la culture scientifique, et définie comme en opposition. Enfin, une dernière caractéristique me semble à souligner : liée au statut du socle commun, la culture humaniste se définit par une liste de connaissances empruntées à diverses disciplines scolaires (français, histoire-géographique, arts plastiques, musique, etc.), mais qui sont reconfigurées dans ce cadre non-disciplinaire des « compétences » du socle. Dans ce contexte, faut-il penser la culture humaniste dans le cadre des disciplines (y compris avec des logiques inter ou transdisciplinaires) ou bien sont-ce au contraire les disciplines qui sont à penser dans le cadre de la culture humaniste? Selon que l'on choisit l'un ou 
l'autre des termes de l'alternative, la réponse en matière d'organisation scolaire et de contenus disciplinaires pourra être sensiblement différente.

\section{En guise de conclusion}

Humanités, culture humaniste: que disent ces mots lourds d'une histoire ancienne et complexe? Que signalent ces références à une tradition scolaire (les humanités) qu'il ne semble pas souhaitable - ni possible - de faire revivre? Ou comment comprendre la résurgence de l'humanisme, sinon comme un mythe, comme le souligne Doquet-Lacoste (2008, p. 100), qui pointe «le risque que ce retour vers l'ancien se fasse au détriment d'autres cheminements créatifs qui se nourrissent du passé pour construire l'avenir»? Les mots sont à prendre avec prudence, mais ils ne sont pas anodins : les humanités telles qu'elles sont actuellement reconstruites dans l'enseignement supérieur n'ont que peu à voir avec les humanités, classiques, modernes voire techniques, qu'a connu l'enseignement secondaire, mais sont sans doute davantage héritière des humanities anglo-saxonnes. Elles permettent de penser une recomposition de disciplines universitaires dominées voire assiégées (lettres classiques ou modernes, philosophie, etc.) qui cherchent à retrouver un second souffle. La situation est assez différente dans l'enseignement primaire et secondaire. Le socle commun et ses piliers (dont celui de la culture humaniste) sont, comme les nombreux dispositifs transversaux qui s'accumulent actuellement, en tension avec les cultures disciplinaires qui ont chacune leurs propres pratiques, contenus, épistémologies, etc. (Cauterman et Daunay, 2010). De ce point de vue, faire de la «culture humaniste» une "compétence» actuelle semble pour le moins paradoxal: quel est cet humanisme décomposable (comme toutes les compétences du socle) en "connaissances ", «capacités " et "attitudes", sinon une version scolaire mythique de l'humanisme, qui l'instrumentalise et qui témoigne par ailleurs d'une forme de nostalgie plutôt réactionnaire?

\section{BIBLIOGRAPHIE}

Balibar, R., (1993). Le colinguisme, Paris, PUF.

Belhoste, B., (1990). «L'enseignement secondaire français et les sciences au début du XXe siècle. La réforme de 1902 des plans d'étude et des programmes ", Revue d'histoire des sciences, tome 43, n ${ }^{\circ} 4$, PUF.

Cauterman, M.-M. et Daunay, B., (2010). « La jungle des dispositifs », Recherches $n^{\circ}$ 52, ARDPF, p. 9-23.

Chervel, A., (2005). « En quoi une culture peut-elle être scolaire? », dans Jacquet-Francillon et Kambouchner (dir.), La crise de la culture scolaire, PUF, p. 77-86.

Chervel, A., (2006). Histoire de l'enseignement du français du XVIIe au XXe siècle, Retz. 
Chervel, A. et Compère, M.-M., (1997). « Les humanités dans l'histoire de l'enseignement français ", Histoire de l'éducation $\mathrm{n}^{\circ}$ 74, Paris, INRP, p. 5-38.

Citton,Y., (2010). L'Avenir des Humanités. Économie de la connaissance ou culture de l'interprétation?, Paris, Éditions de la Découverte.

Compère, M.-M., (1997). « La tardive constitution de l'enseignement des humanités comme objet historique ", Histoire de l'éducation $n^{\circ} 74$, Paris, INRP, p. 187-203.

Denizot, N., (2013). La scolarisation des genres littéraires (1880-2010), Bruxelles, Peter Lang.

Denizot, N. (à paraitre), « Morceaux choisis et patrimonialisation de la littérature (XIXe siècleXXe siècle) ", dans Bishop, M.-F. et Belhadjin, A. (dir.), Les patrimoines littéraires à l'école : tensions et débats actuels, Paris, Honoré Champion.

Doquet-Lacoste, C., (2008). « La culture humaniste, 5e pilier du socle commun des apprentissages : quels objets pour quelles pratiques ? ", in Dubois-Marcoin, D. et Tauveron, C. (dir.), Français, langue et littérature, socle commun [Actes du colloque de Lyon 12-14 mars 2008], Lyon, INRP, p. 97-104.

Doueihi, M., (2011). Pour un humanisme numérique, Paris, Seuil.

Fabiani, J.-L., (2012). « Du chaos des disciplines à la fin de l'ordre disciplinaire ? », Pratiques n 153-154, Littéracies universitaires : nouvelles perspectives, Metz, CRESEF, p. 129-140.

Falucci, C., (1939). L'humanisme dans l'enseignement secondaire, Toulouse, Privat/Paris, Didier.

Houdart-Merot, V. (2012). « Qu'est-ce qu'un classique ? Qu'est-ce qu'une œuvre patrimoniale?», in de Peretti, I. et Ferrier, B. (dir.), Enseigner les « classiques » aujourd'hui, Bruxelles, Peter Lang, p. 23-36.

Huynh, J.-A., (2009). « De la pertinence de l'argumentation dans la construction d'une culture humaniste au XXIe siècle ", Le Français aujourd'hui n¹67, Culture humaniste : textes et pratiques, Paris, Armand Colin/AFEF, p. 53-66.

Jey, M., (1998). La littérature au lycée : Invention d'une discipline (1880-1925), Recherches textuelles $\mathrm{n}^{\circ}$ 3, Université de Metz.

Kahn, P., (2001). « Enseigner les sciences en vue des "usages de la vie". Réflexions sur un paradigme de l'école primaire au XXe siècle, Carrefours de l'éducation n 11, Paris, Armand Colin, p. $34-51$.

Lahire, B., (2008). La raison scolaire. École et pratiques d'écriture, entre savoir et pouvoir, Rennes, PUR.

Laurent, J.-N. et Vignest, R. (dir.), (2010). Enseigner les humanités. Enjeux, programmes et méthodes de la fin du XVIIIe à nos jours, Paris, éditions Kim.

Lelièvre, C., (2009). « Culture humaniste et "socle" de l'école obligatoire », Le français aujourd'hui n ${ }^{\circ} 167$, AFEF-Armand Colin, p. 11-17.

Musset, M., (2008). « De la communale au socle commun : littérature et culture humaniste », Dossier d'actualité de la VST n 33, Lyon, INRP. Disponible en ligne à l'adresse : http://ife.enslyon.fr/vst/DA-Veille/33-fevrier-2008.pdf

Nussbaum, M., (2010). Not for Profit: Why Democracy Needs the Humanities? Princeton University Press.

Pelpel, P. et Troger, V., (2001). Histoire de l'enseignement technique, Paris, Hachette Éducation. 
Pradeau, J.-F., (2013). Les « humanités » au cœur de l'excellence scolaire et professionnelle, Centre d'analyse stratégique. Disponible en ligne à l'adresse : http://www.strategie.gouv.fr/system/ files/130220_-_humanites-dt-v_finaleval_le_25-02.pdf

Savatovsky, D., (1995). « Le français, matière ou discipline ? ", Langages $\mathrm{n}^{\circ} 120$, Les savoirs de la langue : histoire et disciplinarité, Paris, Larousse, p. 52-77.

Simondon, G., Du mode d'existence des objets techniques, Paris, Aubier (22e édition), 1958/2012.

\section{NOTES}

1. Voir par exemple les licences Humanités de Paris X et de l'Université de Strasbourg, les UFR Humanités de Lille 3 et de Bordeaux 3, le département Humanités de L'École Polytechnique, le Centre des Humanités de l'INSA de Lyon, etc.

2. Une redéfinition du socle commun est annoncée, mais non encore publiée au moment de l'écriture de cet article.

3. Décret du 11 juillet 2006. Voir http://www.education.gouv.fr/cid2770/le-socle-commun-deconnaissances-et-de-competences.html

4. Tout au long du XIXe siècle, le terme « humanités » est polysémique à l'école, et une seconde acception - que je signale simplement ici, mais que je n'utiliserai pas - fait des humanités la classe qu'on appelle aussi "poétique ", et qui précède la classe de "rhétorique " (ces deux classes deviendront après 1902 les classes de seconde et de première).

5. A : latin-grec ; B : latin-langues vivantes ; $\mathrm{C}$ : latin-sciences ; D : langues vivantes- sciences.

6. Notamment à la suite des travaux de Gilbert Simondon, pour qui « il y a de la nature humaine dans l'être technique» (1958/2012, p. 248). Sur les humanités techniques, cf. Pelpel et Troger, 2001.

7. Rares sont les emplois qui font d'humanités un simple synonyme d'études classiques, comme dans le rapport de Jean-François Pradeau (2013) pour le Centre d'analyse stratégique, pour qui les « humanités » désignent l'enseignement des langues et des cultures de l'antiquité.

8. Écoles supérieures du professorat et de l'éducation, qui remplacent les ex-IUFM (Institut universitaire de formation des maitres).

9. Je le distingue de « culture humaniste », que je traite infra.

10. Unités de Formation et de Recherche.

11. Pour un tour d'horizon (notamment bibliographique) très complet sur le sujet, cf. Musset, 2008.

12. On trouvera une analyse très intéressante sur le sujet dans Huyhn, 2009.

\section{RÉSUMÉS}

L'article se propose de réfléchir aux «humanités » et à «la culture humaniste " à partir du concept de discipline scolaire : puisque les humanités ne sont pas (plus ?) une discipline, quels rapports entretiennent-elles avec la discipline français, qui est en partie leur héritière, mais qui a également contribué à les redéfinir? Et comment articuler la notion de "culture humaniste » issue du socle commun aux configurations actuelles de la discipline (primaire et secondaire)? 
The article intends to take the discipline of French as a starting point in order to analyse «the humanities» and "humanist culture». Since the humanities are not (no longer?) a discipline, which relationship do they maintain with the discipline of French, which is partly heir to them, but which has also contributed to redefine them. Besides, how is it possible to link the concept of «humanist culture» coming from the «socle commun» to the configurations now prevailing in the discipline as well in primary as in secondary school?

INDEX

Mots-clés : culture humaniste, discipline scolaire, discipline " français », Humanités

Keywords : discipline of French, humanist culture, Humanities, school discipline

\section{AUTEUR}

NATHALIE DENIZOT

Université de Cergy-Pontoise, ÉMA (ÉA 4507) 